\title{
Semi-smooth Points in Some Classical Function Spaces
}

\author{
Beata Derȩgowska, Beata Gryszka, Karol Gryszka, and \\ Paweł Wójcik
}

\begin{abstract}
The investigations of the smooth points in the spaces of continuous function were started by Banach in 1932 considering function space $\mathcal{C}(\Omega)$. Singer and Sundaresan extended the result of Banach to the space of vector valued continuous functions $\mathcal{C}(\mathcal{T}, E)$, where $\mathcal{T}$ is a compact metric space. The aim of this paper is to present a description of semi-smooth points in spaces of continuous functions $\mathcal{C}_{0}(\mathcal{T}, E)$ (instead of smooth points). Moreover, we also find necessary and sufficient condition for semi-smoothness in the general case.
\end{abstract}

Mathematics Subject Classification. 46B20, 46C50, 46B25.

Keywords. Normed space, smoothness, semi-smoothness, function space, norm derivatives.

\section{Introduction}

Smoothness in Banach space has been intensively studied for decades from many different points of view. We refer to the monographs $[2,6]$ and the papers $[10,11]$ for motivations, history, various aspects and problems connected with the subject.

In this paper, motivated by the results published by Banach [2], Singer [10], Sundaresan [11] and Miličić [8,9], we study the notion of semi-smooth points in Banach spaces of continuous functions.

It is necessary to mention a classical result: if $\Omega$ is a compact Hausdorff space, then 


$$
\text { smooth } \begin{aligned}
(\mathcal{C}(\Omega)) & =\left\{f \in \mathcal{C}(\Omega): \text { there exists } t_{o} \text { such that }\|f\|\right. \\
& \left.=\left|f\left(t_{o}\right)\right|>|f(t)| \text { for } t \neq t_{o}\right\}
\end{aligned}
$$

(cf. Banach classical monograph [2, p. 168]). Singer [10] characterized the smooth points of the unit ball of $\mathcal{C}([0,1], E)$, the space of continuous functions with values in the Banach space $E$. This was extended by Sundaresan [11] to the space of vector valued continuous functions $\mathcal{C}(\mathcal{T}, E)$, where $\mathcal{T}$ is a compact metric space.

The main object of this paper is to study the semi-smooth points of the unit ball of $\mathcal{C}_{0}(\mathcal{T}, E)$, when $\mathcal{T}$ is a locally compact topological space. Moreover, in this paper, we find necessary and sufficient condition for semi-smoothness.

It is worth mentioning that in some cases the notion of semi-smoothness may be more convenient than the notion of smoothness. Indeed, recently the concept of semi-smoothness played a significant role in the paper [12], where the notion of semi-smooth points was helpful to solve the open problem posed in 2010 .

\section{Preliminaries}

The paper is organized as follows. In Sect. 3 of the paper, we discuss semismoothness in the space $\mathcal{C}_{0}(\mathcal{T}, E)$. In the Sect. 4 we study semi-smooth points and relate them to the set of supporting functionals.

Before stating the results, we recall some definitions and introduce our notation.

\subsection{Norm Derivatives}

Let $(X,\|\cdot\|)$ be a real normed space. We define norm derivatives:

$\rho_{ \pm}^{\prime}(x, y):=\lim _{\lambda \rightarrow 0^{ \pm}} \frac{\|x+\lambda y\|^{2}-\|x\|^{2}}{2 \lambda}=\|x\| \cdot \lim _{\lambda \rightarrow 0^{ \pm}} \frac{\|x+\lambda y\|-\|x\|}{\lambda}, \quad x, y \in X$.

Convexity of the norm yields that the above definition is meaningful. The mappings $\rho_{+}^{\prime}$ and $\rho_{-}^{\prime}$ are sometimes called the superior and inferior semi inner products and their following properties can be found, e.g., in $[1,7]$ :

$$
\begin{aligned}
& \text { (nd1) } \forall_{x, y \in X} \forall_{\alpha \in \mathbb{R}} \quad \rho_{ \pm}^{\prime}(x, \alpha x+y)=\alpha\|x\|^{2}+\rho_{ \pm}^{\prime}(x, y) \text {; } \\
& \text { (nd2) } \forall_{x, y \in X} \forall_{\alpha \geq 0} \quad \rho_{ \pm}^{\prime}(\alpha x, y)=\alpha \rho_{ \pm}^{\prime}(x, y)=\rho_{ \pm}^{\prime}(x, \alpha y) \text {; } \\
& \text { (nd3) } \forall_{x, y \in X} \forall_{\alpha<0} \quad \rho_{ \pm}^{\prime}(\alpha x, y)=\alpha \rho_{\mp}^{\prime}(x, y)=\rho_{ \pm}^{\prime}(x, \alpha y) \text {; } \\
& \text { (nd4) } \quad \forall_{x \in X} \quad \rho_{ \pm}^{\prime}(x, x)=\|x\|^{2} \text {; } \\
& \text { (nd5) } \quad \forall_{x, y \in X} \quad\left|\rho_{ \pm}^{\prime}(x, y)\right| \leq\|x\| \cdot\|y\| \text {; } \\
& \text { (nd6) } \quad \forall_{x, y \in X} \quad \rho_{-}^{\prime}(x, y) \leq \rho_{+}^{\prime}(x, y) \text {. }
\end{aligned}
$$

Moreover, mappings $\rho_{+}^{\prime}, \rho_{-}^{\prime}$ are continuous with respect to the second variable, but not necessarily with respect to the first one. Note that if $(X,\langle\cdot \mid-\rangle)$ is an inner product space, then $\langle y \mid x\rangle=\rho_{+}^{\prime}(x, y)=\rho_{-}^{\prime}(x, y)$ for arbitrary $x, y \in X$. 
Miličić introduced (see [8]) the following concept. Let us define $\rho^{\prime}: X \times$ $X \rightarrow \mathbb{R}$ by

$$
\rho^{\prime}(x, y):=\frac{1}{2}\left(\rho_{-}^{\prime}(x, y)+\rho_{+}^{\prime}(x, y)\right), \quad x, y \in X .
$$

The above functional $\rho^{\prime}$ is also denoted by $\langle y \mid x\rangle_{g}$ and called an $M$-semi inner product - cf. Miličić [9] and also [7]. From the above properties of the mappings $\rho_{ \pm}^{\prime}$ we get:

(Msip1) $\quad \forall_{x, y \in X} \forall_{\alpha \in \mathbb{R}} \quad \rho^{\prime}(x, \alpha x+y)=\alpha\|x\|^{2}+\rho^{\prime}(x, y) ;$

(Msip2) $\quad \forall_{x, y \in X} \quad \forall_{\alpha \in \mathbb{R}} \quad \rho^{\prime}(\alpha x, y)=\alpha \rho^{\prime}(x, y)=\rho^{\prime}(x, \alpha y)$;

(Msip3) $\quad \forall_{x \in X} \quad \rho^{\prime}(x, x)=\|x\|^{2}$;

(Msip4) $\quad \forall_{x, y \in X} \quad\left|\rho^{\prime}(x, y)\right| \leq\|x\| \cdot\|y\|$.

As a consequence of (nd6) we have

$$
\rho_{-}^{\prime}(x, y) \leq \rho^{\prime}(x, y) \leq \rho_{+}^{\prime}(x, y), \quad x, y \in X .
$$

Moreover, $\rho^{\prime}$ is continuous with respect to the second variable.

It is worth mentioning that $M$-semi inner product $\rho^{\prime}$ was recently very helpful to extended results concerning Birkhoff-James orthogonally additive mappings; in particular, see [3, Theorem 3.3].

The unit sphere of $X$ is denoted by $S(X)$. Fix $x \in X \backslash\{0\}$. We consider the set $J(x)$ defined as follows

$$
J(x):=\left\{x^{*} \in X^{*}:\left\|x^{*}\right\|=1,\left\|x^{*}(x)\right\|=\|x\|\right\} .
$$

It is easy to check that the set $J(x)$ is convex, closed and $J(x) \subseteq S\left(X^{*}\right)$. By the Hahn-Banach theorem we get $J(x) \neq \emptyset$ for all $x \in X \backslash\{0\}$. Moreover, it is easy to see that $J(\alpha x)=J(x)$ for all $\alpha \in \mathbb{R} \backslash\{0\}, x \in X \backslash\{0\}$.

Let us recall the following result containing a representation of the norm derivatives $\rho_{ \pm}^{\prime}$ in terms of supporting functionals.

Theorem 2.1. ([7, p. 36]). Let $X$ be a real normed space. Then one has the representation:

$$
\begin{aligned}
& \rho_{-}^{\prime}(x, y)=\|x\| \cdot \inf \left\{x^{*}(y): x^{*} \in J(x)\right\} \text { and } \\
& \rho_{+}^{\prime}(x, y)=\|x\| \cdot \sup \left\{x^{*}(y): x^{*} \in J(x)\right\} \text { for all } x, y \in X .
\end{aligned}
$$

So, in particular,

$$
\forall_{x^{*} \in J(x)} \quad \rho_{-}^{\prime}(x, y) \leq\|x\| \cdot x^{*}(y) \leq \rho_{+}^{\prime}(x, y) .
$$

\subsection{Smoothness and Semi-smoothness}

From now on we assume that the considered normed spaces are real and their dimensions are not less than 2 . Let $(X,\|\cdot\|)$ be a normed space over $\mathbb{R}$. We say that $X$ is smooth at point $x_{o}$ if $J(x)$ contains a unique element in $X^{*}$. Now, we consider a set

$$
\mathcal{N}_{s m}(X):=\{x \in X: X \text { is smooth at } x\} \cup\{0\} .
$$


It will be helpful to reformulate the definition of smoothness as follows:

$$
x \in \mathcal{N}_{\text {sm }}(X) \Leftrightarrow \operatorname{card} J(x)=1 .
$$

If $X$ is a separable real Banach space, then $\mathcal{N}_{s m}(X)$ is dense. We say that a normed space $X$ is smooth if $X=\mathcal{N}_{s m}(X)$.

Now we will give a characterization of smoothness at a point in terms of the norm derivatives (see $[1,7]$ ).

Theorem 2.2. ([1,7]). Let $X$ be a real normed space and let $x_{o} \in X \backslash\{0\}$. Then the following statements are equivalent:

(a) $X$ is smooth at $x_{o}$, i.e., $x_{o} \in \mathcal{N}_{\text {sm }}(X)$;

(b) $\rho_{-}^{\prime}\left(x_{o}, \cdot\right)$ is linear;

(c) $\rho_{+}^{\prime}\left(x_{o}, \cdot\right)$ is linear;

(d) $\rho_{-}^{\prime}\left(x_{o}, \cdot\right) \in X^{*}$;

(e) $\rho_{+}^{\prime}\left(x_{o}, \cdot\right) \in X^{*}$;

(f) $\forall_{x, y \in X} \quad \rho_{-}^{\prime}\left(x_{o}, y\right)=\rho_{+}^{\prime}\left(x_{o}, y\right)$.

It is clear that if $X$ is smooth, then the $M$-semi-inner product in the sense of Miličić $\rho^{\prime}(\diamond, \cdot)$ is linear in the second argument. However, there also exists non-smooth spaces from which the mapping $\rho^{\prime}(\diamond, \cdot)$ is linear in the second variable too ([8, Example 8.1], [7, p. 51]).

A real normed space $X$ is called semi-smooth if $\rho^{\prime}$ is additive (or, equivalently, linear; see (Msip2)) with respect to the second variable, i.e.,

$$
\rho^{\prime}(x, y+z)=\rho^{\prime}(x, y)+\rho^{\prime}(x, z), \quad x, y, z \in X .
$$

Each smooth space is semi-smooth in the above sense but not conversely $\left(l^{1}\right.$ is a suitable example; see [8, Example 8.1] and [7, p. 51]).

The notion of semi-smooth spaces motivates this paper. We say that a normed space $(X,\|\cdot\|)$ is semi-smooth at the point $x_{o} \in X$ if it satisfies

$$
\forall y, z \in X \quad \rho^{\prime}\left(x_{o}, y+z\right)=\rho^{\prime}\left(x_{o}, y\right)+\rho^{\prime}\left(x_{o}, z\right),
$$

or, equivalently, if the mapping $\rho^{\prime}\left(x_{o}, \cdot\right): X \rightarrow \mathbb{R}$ is linear. As was mentioned, semi-smooth points played a crucial role in [12].

Similarly as above, we define

$$
\mathcal{N}_{s m}^{s}(X):=<\{x \in X: X \text { is semi-smooth at } x\} .
$$

Thus we can write $X=\mathcal{N}_{s m}^{s}(X)$ if and only if $X$ is semi-smooth. We observe that

$$
\mathcal{N}_{s m}(X) \subseteq \mathcal{N}_{s m}^{s}(X) .
$$

Indeed, let $x \in \mathcal{N}_{s m}(X)$. Then for all $y \in X$ we have $\rho_{-}^{\prime}(x, y)=\rho_{+}^{\prime}(x, y)$. Applying (2.1) and $(2.2)$ we get $\rho^{\prime}(x, \cdot)=\rho_{-}^{\prime}(x, \cdot)=\rho_{+}^{\prime}(x, \cdot)=\|x\| \cdot x^{*}(\cdot)$ for all $x^{*} \in J(x)$, hence $\rho^{\prime}(x, \cdot)$ is linear, and therefore $x \in \mathcal{N}_{s m}^{s}(X)$. As we know already from $\left[8\right.$, Example 8.1], [7, p. 51], we have $\mathcal{N}_{s m}(X)\left(l^{1}\right) \varsubsetneqq \mathcal{N}_{s m}^{s}(X)\left(l^{1}\right)$.

We summarize our observations in the following simple result. 
Theorem 2.3. Let $X$ be a real normed space and let $x_{o} \in X \backslash\{0\}$. Then the following statements are equivalent:

(a) $X$ is semi-smooth at $x_{o}$, i.e., $x_{o} \in \mathcal{N}_{s m}^{s}(X)$;

(b) $\rho^{\prime}\left(x_{o}, \cdot\right)$ is additive;

(c) $\rho^{\prime}\left(x_{o}, \cdot\right)$ is linear;

(d) $\rho^{\prime}\left(x_{o}, \cdot\right) \in X^{*}$;

(e) $X$ is semi-smooth at $\alpha x_{o}$ for all $\alpha \in \mathbb{R} \backslash\{0\}$.

In the papers $[4,5], \rho$ and $\rho_{ \pm}$-orthogonalities were considered as well as other characterizations of semi-smooth spaces in terms of approximately $\rho, \rho_{ \pm^{-}}$ orthogonalities.

\section{Semi-smoothness in Spaces of Continuous Functions}

In this section we discuss the notion of semi-smoothness in continuous function spaces.

If $\mathcal{T}$ is a locally compact Hausdorff space and $E$ a real normed linear space, we let $\mathcal{C}_{0}(\mathcal{T}, E)$ denote the space of all continuous functions $f$ from $\mathcal{T}$ to $E$ which vanish at infinity (namely, a function $f$ vanishes at infinity if for every $\varepsilon>0$ there is a compact set $K \subseteq \mathcal{T}$ such that $\|f(t)\|_{E}<\varepsilon$ if $\left.t \in \mathcal{T} \backslash K\right)$ with $\|f\|_{\infty}:=\sup \left\{\|f(t)\|_{E}: t \in \mathcal{T}\right\}$. In this section we want to focus our attention on $\mathcal{C}_{0}(\mathcal{T}, E)$. We denote $\mathcal{M}(f):=\left\{t \in \mathcal{T}:\|f(t)\|_{E}=\|f\|_{\infty}\right\}$.

Lemma 3.1. For any $f \in \mathcal{C}_{0}(\mathcal{T}, E)$ the set $\mathcal{M}(f)$ is not empty and compact.

Proof. Take $f \neq 0$ and consider $\varepsilon=\frac{\|f\|_{\infty}}{2}$. Take a compact set $K \subseteq \mathcal{T}$ such that $\|f(t)\|_{E}<\varepsilon$ if $t \in \mathcal{T} \backslash K$. We know that the mapping $K \ni t \mapsto\|f(t)\|_{E} \in$ $\left[0,\|f\|_{\infty}\right] \subseteq \mathbb{R}$ is continuous and $K$ is compact. Therefore this mapping attains its maximum, i.e., there is $t_{0} \in K$ such that $\|f\|_{\infty}=\left\|f\left(t_{0}\right)\right\|_{E}$. Thus the set $M(f)$ is not empty. Moreover, it is easy to check that the set $M(f)$ is closed. Therefore it is compact.

The next theorem will be useful for the study of semi-smooth points. The same symbol, $\rho_{+}^{\prime}$, will be used to denote the norm derivatives on $E$ and on $\mathcal{C}_{0}(\mathcal{T}, E)$.

Theorem 3.2. ([13, Theorem 20.8, p. 462]). Let $\mathcal{T}$ be a locally compact Hausdorff. Let $E$ be a real normed space. Assume that $f, g \in \mathcal{C}_{0}(\mathcal{T}, E) \backslash\{0\}$. Then

$$
\begin{aligned}
& \rho_{+}^{\prime}(f, g)=\max \left\{\rho_{+}^{\prime}(f(t), g(t)): t \in \mathcal{M}(f)\right\}, \\
& \rho_{-}^{\prime}(f, g)=\min \left\{\rho_{-}^{\prime}(f(t), g(t)): t \in \mathcal{M}(f)\right\} .
\end{aligned}
$$

Now we prove the first main result of this section.

Theorem 3.3. Suppose that $\mathcal{T}$ is a locally compact space. Let $E$ be a real normed space. Assume that $f \in \mathcal{C}_{0}(\mathcal{T}, E), f \neq 0$ and let $\operatorname{card} \mathcal{M}(f)=1$, i.e., $\mathcal{M}(f)=\left\{t_{1}\right\}$. The following statements are equivalent:
(A) $f$ is a semi-smooth point, i.e., $f \in \mathcal{N}_{s m}^{s}\left(\mathcal{C}_{0}(\mathcal{T}, E)\right)$,
(B) $f\left(t_{1}\right)$ is a semi-smooth point, i.e., $f\left(t_{1}\right) \in \mathcal{N}_{s m}^{s}(E)$. 
Proof. By (3.1) we have

$$
\begin{aligned}
\rho^{\prime}(f, g) & =\frac{1}{2}\left(\rho_{-}^{\prime}(f, g)+\rho_{+}^{\prime}(f, g)\right)= \\
& =\frac{1}{2}\left(\rho_{-}^{\prime}\left(f\left(t_{1}\right), g\left(t_{1}\right)\right)+\rho_{+}^{\prime}\left(f\left(t_{1}\right), g\left(t_{1}\right)\right)\right)= \\
& =\rho^{\prime}\left(f\left(t_{1}\right), g\left(t_{1}\right)\right) .
\end{aligned}
$$

Therefore

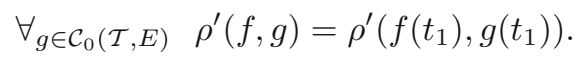

We prove $(\mathrm{A}) \Rightarrow(\mathrm{B})$. Suppose $f \in \mathcal{N}_{s m}^{s}\left(\mathcal{C}_{0}(\mathcal{T}, E)\right)$. By Theorem 2.3, we may assume that $\|f\|_{\infty}=1$, and then $\left\|f\left(t_{1}\right)\right\|_{E}=1$. Fix $x, y$ in $E \backslash\{0\}$. The functions defined by $g(t):=\|f(t)\|_{E} \cdot x, h(t):=\|f(t)\|_{E} \cdot y$ are continuous and $g, h \in \mathcal{C}_{0}(\mathcal{T}, E)$. Then, using (3.2) again we deduce

$$
\begin{aligned}
\rho^{\prime}\left(f\left(t_{1}\right), x+y\right) & =\rho^{\prime}\left(f\left(t_{1}\right), g\left(t_{1}\right)+h\left(t_{1}\right)\right) \stackrel{(3.2)}{=} \rho^{\prime}(f, g+h) \stackrel{(\mathrm{A})}{=} \\
& =\rho^{\prime}(f, g)+\rho^{\prime}(f, h) \stackrel{(3.2)}{=} \rho^{\prime}\left(f\left(t_{1}\right), g\left(t_{1}\right)\right)+\rho^{\prime}\left(f\left(t_{1}\right), h\left(t_{1}\right)\right)= \\
& =\rho^{\prime}\left(f\left(t_{1}\right), y\right)+\rho^{\prime}\left(f\left(t_{1}\right), y\right),
\end{aligned}
$$

which means that $f\left(t_{1}\right) \in \mathcal{N}_{s m}^{s}(E)$. On the other hand, from (3.2) it is very easy to prove that $(\mathrm{B}) \Rightarrow(\mathrm{A})$.

A topological space is called perfectly normal (or $T_{6}$ ) if it is normal and every closed subset is a $G_{\delta}$ subset. The next result is from topology. It may be known to the reader, but it is presented here for the convenience.

Theorem 3.4. (Vedenisov). In a perfectly normal (i.e., $T_{6}$ ) topological space $(X, \tau)$ there is for each pair $F, H$ of disjoint closed sets a continuous function $f: X \rightarrow[0,1]$ that is 0 on $F$ and 1 on $H$ and $0<f(t)<1$ for $t \in X \backslash(F \cup H)$.

Of more immediate interest to us are the facts that metric spaces are perfectly normal spaces. Now we prove the second main result of this section.

Theorem 3.5. Suppose that $\mathcal{T}$ is a locally compact perfectly normal space. Let $E$ be a real normed space. Assume that $f \in \mathcal{C}_{0}(\mathcal{T}, E), f \neq 0$ and let $\operatorname{card} \mathcal{M}(f) \geq 3$. Then $f$ is not a semi-smooth point, i.e., $f \notin \mathcal{N}_{s m}^{s}\left(\mathcal{C}_{0}(\mathcal{T}, E)\right)$.

Proof. There exist $t_{1}, t_{2}, t_{3} \in \mathcal{M}(f)$ such that $t_{1} \neq t_{2} \neq t_{3} \neq t_{1}$. Using the Vedenisov Extension Theorem (Theorem 3.4) twice, we are able to find a function $\gamma \in \mathcal{C}(\mathcal{T}, \mathbb{R})$ such that $\gamma\left(t_{1}\right)=1, \gamma\left(t_{2}\right)=-1, \gamma\left(t_{3}\right)=0$, and $\gamma\left(t_{2}\right)<\gamma(t)<\gamma\left(t_{1}\right)$ for all $t \in \mathcal{T} \backslash\left\{t_{1}, t_{2}\right\}$.

Using again the Vedenisov Extension Theorem, we are able to find a function $\eta \in \mathcal{C}(\mathcal{T}, \mathbb{R})$ such that $\eta\left(t_{1}\right)=0, \eta\left(t_{2}\right)=-1, \eta\left(t_{3}\right)=1$, and $\eta\left(t_{2}\right)<$ $\eta(t)<\eta\left(t_{3}\right)$ for all $t \in \mathcal{T} \backslash\left\{t_{2}, t_{3}\right\}$.

Let us define $g, h: \mathcal{T} \rightarrow E$ by $g:=\gamma \cdot f, h:=\eta \cdot f$. Since $\gamma, \eta \in \mathcal{C}_{0}(\mathcal{T}, \mathbb{R})$, $|\gamma(t)| \leq 1,|\eta(t)| \leq 1$ (for all $t \in \mathcal{T}$ ) and $f \in \mathcal{C}_{0}(\mathcal{T}, E)$, we have $g, h \in \mathcal{C}_{0}(\mathcal{T} ; E)$. 
Thus we obtain

$$
\begin{aligned}
& \rho_{+}^{\prime}(f, g) \stackrel{(3.1)}{=} \quad \max \left\{\rho_{+}^{\prime}(f(t), g(t)): t \in \mathcal{M}(f)\right\} \\
& =\max \left\{\rho_{+}^{\prime}(f(t), \gamma(t) \cdot f(t)): t \in \mathcal{M}(f)\right\} \\
& \stackrel{(\mathrm{nd} 2, \mathrm{nd} 3)}{=} \max \left\{\gamma(t) \cdot \rho_{ \pm}^{\prime}(f(t), f(t)): t \in \mathcal{M}(f)\right\} \\
& \stackrel{(\text { nd4) }}{=} \quad \max \left\{\gamma(t) \cdot\|f(t)\|_{E}^{2}: t \in \mathcal{M}(f)\right\} \\
& =\max \left\{\gamma(t) \cdot\|f\|_{\infty}^{2}: t \in \mathcal{M}(f)\right\}=\gamma\left(t_{1}\right) \cdot\|f\|_{\infty}^{2}=\|f\|_{\infty}^{2} .
\end{aligned}
$$

Similarly, using (3.1) and (nd2-4) one gets $\rho_{-}^{\prime}(f, g)=-\|f\|_{\infty}^{2}$ and consequently,

$$
\rho^{\prime}(f, g)=\frac{1}{2}\left(\rho_{+}^{\prime}(f, g)+\rho_{-}^{\prime}(f, g)\right)=\frac{1}{2}\left(\|f\|_{\infty}^{2}-\|f\|_{\infty}^{2}\right)=0 .
$$

In a similar way one can prove: $\rho_{+}^{\prime}(f, h)=\|f\|_{\infty}^{2}, \rho_{-}^{\prime}(f, h)=-\|f\|_{\infty}^{2}$. Hence

$$
\rho^{\prime}(f, h)=0 .
$$

Since $g+h=(\gamma+\eta) \cdot f$, it is easy to verify that

$$
g\left(t_{1}\right)+h\left(t_{1}\right)=f\left(t_{1}\right), \quad g\left(t_{2}\right)+h\left(t_{2}\right)=-2 f\left(t_{2}\right), \quad g\left(t_{3}\right)+h\left(t_{3}\right)=f\left(t_{3}\right) .
$$

In the same manner, one can prove $\rho_{-}^{\prime}(f, g+h)=-2\|f\|_{\infty}^{2}$. It is easy to verify that $\gamma(t)+\eta(t)<2$ for all $t \in \mathcal{M}(f)$. Since $\mathcal{M}(f)$ is compact (see Lemma $3.1)$, there is $\alpha \in(0,2)$ such that $\gamma(t)+\eta(t) \leq \alpha$ for all $t \in \mathcal{M}(f)$. Since $g+h=(\gamma+\eta) \cdot f$, it follows that

$$
\begin{aligned}
\rho_{+}^{\prime}(f, g+h) & \stackrel{(3.1)}{=} \max \left\{\rho_{+}^{\prime}(f(t),(\gamma(t)+\eta(t)) \cdot f(t)): t \in \mathcal{M}(f)\right\} \\
\left(\stackrel{\mathrm{nd} 2, \mathrm{nd} 3)}{=} \max \left\{(\gamma(t)+\eta(t)) \cdot \rho_{ \pm}^{\prime}(f(t), f(t)): t \in \mathcal{M}(f)\right\}\right. & \operatorname{(nd4)}=\|f\|_{\infty}^{2} \cdot \max \{(\gamma(t)+\eta(t)): t \in \mathcal{M}(f)\} \leq\|f\|_{\infty}^{2} \cdot \alpha
\end{aligned}
$$

and

$$
\begin{aligned}
\rho^{\prime}(f, g+h) & =\frac{1}{2}\left(\rho_{-}^{\prime}(f, g+h)+\rho_{+}^{\prime}(f, g+h)\right)=\frac{1}{2}\left(-2\|f\|_{\infty}^{2}+\rho_{+}^{\prime}(f, g+h)\right) \\
& \leq \frac{1}{2}\left(-2\|f\|_{\infty}^{2}+\alpha \cdot\|f\|_{\infty}^{2}\right)<\frac{1}{2}\left(-2\|f\|_{\infty}^{2}+2\|f\|_{\infty}^{2}\right)=0 .
\end{aligned}
$$

Thus by (3.3), (3.4) and (3.5), we have $\rho^{\prime}(f, g+h) \neq \rho^{\prime}(f, g)+\rho^{\prime}(f, h)$. This means that $f \notin \mathcal{N}_{s m}^{s}(\mathcal{C}(\mathcal{T} ; E))$.

We are going to present the third main results of this section. We will need a simple lemma.

Lemma 3.6. Let $X$ be a real normed space and let $x_{o} \in X \backslash\{0\}$. Then the following statements are equivalent:

(i) $X$ is smooth at $x_{o}$, i.e., $x_{o} \in \mathcal{N}_{s m}(X)$;

(ii) $\forall_{y, z \in X} \quad\|y\| \leq 1,\|z\| \leq 1 \quad \Rightarrow \quad \rho_{-}^{\prime}\left(x_{o}, y+z\right)=\rho_{-}^{\prime}\left(x_{o}, y\right)+\rho_{-}^{\prime}\left(x_{o}, z\right)$;

(iii) $\forall_{y, z \in X} \quad\|y\|=\|z\| \Rightarrow \rho_{-}^{\prime}\left(x_{o}, y+z\right)=\rho_{-}^{\prime}\left(x_{o}, y\right)+\rho_{-}^{\prime}\left(x_{o}, z\right)$. 
Proof. Assume condition (i). It follows from Theorem $2.2((\mathrm{a}) \Leftrightarrow(\mathrm{b}) \Leftrightarrow(\mathrm{d}))$ that (ii) and (iii) holds.

For the proof of (iii) $\Longrightarrow($ i) take any $y \neq 0$. It follows from (iii) that

$$
0=\rho_{-}^{\prime}\left(x_{o}, y+(-y)\right)=\rho_{-}^{\prime}\left(x_{o}, y\right)+\rho_{-}^{\prime}\left(x_{o},-y\right) \text {. }
$$

Using (3.6) and (nd3) we have

$$
\rho_{-}^{\prime}\left(x_{o}, y\right)=-\rho_{-}^{\prime}\left(x_{o},-y\right)=\rho_{+}^{\prime}\left(x_{o}, y\right)
$$

and thus $\rho_{-}^{\prime}\left(x_{o}, \cdot\right)=\rho_{+}^{\prime}\left(x_{o}, \cdot\right)$. By Theorem 2.2 we have $x_{o} \in \mathcal{N}_{s m}(X)$.

The proof of (ii) $\Longrightarrow$ (i) is similar: we fix $y$ with $\|y\| \leq 1$ and follow the argument to obtain equation (3.7). Then by (nd2) we get that $\rho_{-}^{\prime}\left(x_{o}, y\right)=$ $\rho_{+}^{\prime}\left(x_{o}, y\right)$ for all $y \in Y$. Thus $x_{o} \in \mathcal{N}_{s m}(X)$.

We are now in a position to prove the third main results of this section.

Theorem 3.7. Suppose that $\mathcal{T}$ is a locally compact space. Let $E$ be a real normed space. Assume that $f \in \mathcal{C}_{0}(\mathcal{T}, E), f \neq 0$ and let $\operatorname{card} \mathcal{M}(f)=2$, i.e., $\mathcal{M}(f)=\left\{t_{1}, t_{2}\right\}$. The following statements are equivalent:

(A) $f$ is a semi-smooth point, i.e., $f \in \mathcal{N}_{s m}^{s}\left(\mathcal{C}_{0}(\mathcal{T}, E)\right)$,

(B) $f\left(t_{1}\right), f\left(t_{2}\right)$ are smooth points, i.e., $f\left(t_{1}\right), f\left(t_{2}\right) \in \mathcal{N}_{\text {sm }}(E)$.

Proof. We start with proving $(\mathrm{B}) \Rightarrow(\mathrm{A})$. We assume that $\mathcal{M}(f)=\left\{t_{1}, t_{2}\right\}$. Fix arbitrarily $g \in \mathcal{C}_{0}(\mathcal{T}, E)$ such that $g \neq 0$. From Theorem 3.2 we have

$$
\rho_{+}^{\prime}(f, g)=\rho_{+}^{\prime}\left(f\left(t_{1}\right), g\left(t_{1}\right)\right) \quad \text { and } \quad \rho_{-}^{\prime}(f, g)=\rho_{-}^{\prime}\left(f\left(t_{2}\right), g\left(t_{2}\right)\right),
$$

or

$$
\rho_{+}^{\prime}(f, g)=\rho_{+}^{\prime}\left(f\left(t_{2}\right), g\left(t_{2}\right)\right) \quad \text { and } \quad \rho_{-}^{\prime}(f, g)=\rho_{-}^{\prime}\left(f\left(t_{1}\right), g\left(t_{1}\right)\right) \text {. }
$$

Since both $f\left(t_{1}\right)$ and $f\left(t_{2}\right)$ are smooth points, we have

$$
\begin{aligned}
& \rho_{-}^{\prime}\left(f\left(t_{1}\right), g\left(t_{1}\right)\right)=\rho_{+}^{\prime}\left(f\left(t_{1}\right), g\left(t_{1}\right)\right) \text { and } \\
& \rho_{-}^{\prime}\left(f\left(t_{2}\right), g\left(t_{2}\right)\right)=\rho_{+}^{\prime}\left(f\left(t_{2}\right), g\left(t_{2}\right)\right) .
\end{aligned}
$$

From (3.8), (3.9), (3.10) we have

$$
\begin{aligned}
\rho^{\prime}(f, g) & =\frac{1}{2}\left(\rho_{+}^{\prime}(f, g)+\rho_{-}^{\prime}(f, g)\right)= \\
& =\frac{1}{2}\left(\rho_{+}^{\prime}\left(f\left(t_{1}\right), g\left(t_{1}\right)\right)+\rho_{+}^{\prime}\left(f\left(t_{2}\right), g\left(t_{2}\right)\right)\right)
\end{aligned}
$$

for all $g \in \mathcal{C}_{0}(\mathcal{T}, E)$. 
Fix arbitrarily two functions $h, k \in \mathcal{C}_{0}(\mathcal{T}, E)$. Then we have

$$
\begin{gathered}
\rho^{\prime}(f, h+k) \stackrel{(3.11)}{=} \frac{1}{2}\left(\rho_{+}^{\prime}\left(f\left(t_{1}\right), h\left(t_{1}\right)+k\left(t_{1}\right)\right)+\rho_{+}^{\prime}\left(f\left(t_{2}\right), h\left(t_{2}\right)+k\left(t_{2}\right)\right)\right) \\
\left(\begin{array}{c}
f\left(t_{1}\right), f\left(t_{2}\right) \\
\in \mathcal{N}_{s m}(E) \\
=
\end{array}\right) \frac{1}{2}\left(\rho_{+}^{\prime}\left(f\left(t_{1}\right), h\left(t_{1}\right)\right)+\rho_{+}^{\prime}\left(f\left(t_{1}\right), k\left(t_{1}\right)\right)+\right. \\
\left.\quad+\rho_{+}^{\prime}\left(f\left(t_{2}\right), h\left(t_{2}\right)\right)+\rho_{+}^{\prime}\left(f\left(t_{2}\right), k\left(t_{2}\right)\right)\right) \\
=\quad \frac{1}{2}\left(\rho_{+}^{\prime}\left(f\left(t_{1}\right), h\left(t_{1}\right)\right)+\rho_{+}^{\prime}\left(f\left(t_{2}\right), h\left(t_{2}\right)\right)\right)+ \\
\quad+\frac{1}{2}\left(\rho_{+}^{\prime}\left(f\left(t_{1}\right), k\left(t_{1}\right)\right)+\rho_{+}^{\prime}\left(f\left(t_{2}\right), k\left(t_{2}\right)\right)\right) \\
\stackrel{(3.11)}{=} \quad \rho^{\prime}(f, h)+\rho^{\prime}(f, k) .
\end{gathered}
$$

which means that $f \in \mathcal{N}_{s m}^{s}(\mathcal{C}(\mathcal{T}, E))$.

For the proof of $(\mathrm{A}) \Rightarrow(\mathrm{B})$ fix arbitrarily two vectors $y, z \in E$ such that $\|y\| \leq 1,\|z\| \leq 1$. In order to show that $f\left(t_{1}\right) \in \mathcal{N}_{s m}(E)$, we use Lemma 3.6. By Urysohn's Lemma there is a continuous function $\varphi: \mathcal{T} \rightarrow[0,1]$ such that $\varphi\left(t_{1}\right)=0, \varphi\left(t_{2}\right)=1$.

Let us introduce the functions $g, h$ from $\mathcal{T}$ into $E$ defined by

$$
\begin{aligned}
& g(t):=\|f(t)\|_{E} \cdot(1-\varphi(t)) \cdot y+\varphi(t) \cdot f(t), \\
& h(t):=\|f(t)\|_{E} \cdot(1-\varphi(t)) \cdot z+\varphi(t) \cdot f(t) .
\end{aligned}
$$

It is a straightforward verification to show that $g, h \in \mathcal{C}(\mathcal{T}, E)$ and $\|g\|_{\infty} \leq$ $\|f\|_{\infty},\|h\|_{\infty} \leq\|f\|_{\infty}$. Moreover,

$$
g\left(t_{1}\right)=\|f\|_{\infty} y, \quad g\left(t_{2}\right)=f\left(t_{2}\right), \quad h\left(t_{1}\right)=\|f\|_{\infty} z, \quad h\left(t_{2}\right)=f\left(t_{2}\right) .
$$

We have

$$
\begin{array}{ll}
\rho_{+}^{\prime}\left(f\left(t_{1}\right),\|f\|_{\infty} y\right)=\rho_{+}^{\prime}\left(f\left(t_{1}\right), g\left(t_{1}\right)\right)=: \alpha, & \rho_{+}^{\prime}\left(f\left(t_{2}\right), g\left(t_{2}\right)\right)=\|f\|_{\infty}^{2}, \\
\rho_{-}^{\prime}\left(f\left(t_{1}\right),\|f\|_{\infty} y\right)=\rho_{-}^{\prime}\left(f\left(t_{1}\right), g\left(t_{1}\right)\right)=: \beta, \quad & \rho_{-}^{\prime}\left(f\left(t_{2}\right), g\left(t_{2}\right)\right)=\|f\|_{\infty}^{2}
\end{array}
$$

and

$$
\begin{array}{ll}
\rho_{+}^{\prime}\left(f\left(t_{1}\right),\|f\|_{\infty} z\right)=\rho_{+}^{\prime}\left(f\left(t_{1}\right), h\left(t_{1}\right)\right)=: \gamma, & \rho_{+}^{\prime}\left(f\left(t_{2}\right), h\left(t_{2}\right)\right)=\|f\|_{\infty}^{2}, \\
\rho_{-}^{\prime}\left(f\left(t_{1}\right),\|f\|_{\infty} z\right)=\rho_{-}^{\prime}\left(f\left(t_{1}\right), h\left(t_{1}\right)\right)=: \delta, & \rho_{-}^{\prime}\left(f\left(t_{2}\right), h\left(t_{2}\right)\right)=\|f\|_{\infty}^{2} .
\end{array}
$$

Combining (nd5) and (3.13), (3.14), we immediately get

$$
|\alpha| \leq\|f\|_{\infty}^{2}, \quad|\beta| \leq\|f\|_{\infty}^{2}, \quad|\gamma| \leq\|f\|_{\infty}^{2}, \quad|\delta| \leq\|f\|_{\infty}^{2} .
$$

Using (3.1), (3.13), (3.14), (3.15) and the equality $M(f)=\left\{t_{1}, t_{2}\right\}$, we obtain

$$
\rho_{+}^{\prime}(f, g)=\|f\|_{\infty}^{2}, \quad \rho_{-}^{\prime}(f, g)=\beta \quad \text { and } \quad \rho_{+}^{\prime}(f, h)=\|f\|_{\infty}^{2}, \quad \rho_{-}^{\prime}(f, h)=\delta,
$$

and hence

$$
\rho^{\prime}(f, g)+\rho^{\prime}(f, h)=\frac{1}{2}\left(\|f\|_{\infty}^{2}+\beta\right)+\frac{1}{2}\left(\|f\|_{\infty}^{2}+\delta\right)=\|f\|_{\infty}^{2}+\frac{1}{2}(\beta+\delta) .
$$


On the other hand, we also consider

$$
\begin{aligned}
& \rho_{+}^{\prime}\left(f\left(t_{1}\right), g\left(t_{1}\right)+h\left(t_{1}\right)\right)=: \varepsilon, \quad \rho_{+}^{\prime}\left(f\left(t_{2}\right), g\left(t_{2}\right)+h\left(t_{2}\right)\right)=2\|f\|_{\infty}^{2}, \\
& \rho_{-}^{\prime}\left(f\left(t_{1}\right), g\left(t_{1}\right)+h\left(t_{1}\right)\right)=: \eta, \quad \rho_{-}^{\prime}\left(f\left(t_{2}\right), g\left(t_{2}\right)+h\left(t_{2}\right)\right)=2\|f\|_{\infty}^{2} .
\end{aligned}
$$

By (3.17) and (nd5) we have

$$
\begin{aligned}
& |\varepsilon|=\left|\rho_{+}^{\prime}\left(f\left(t_{1}\right), g\left(t_{1}\right)+h\left(t_{1}\right)\right)\right| \leq\left\|f\left(t_{1}\right)\right\| \cdot\left\|g\left(t_{1}\right)+h\left(t_{1}\right)\right\| \leq 2\|f\|_{\infty}^{2}, \\
& |\eta|=\left|\rho_{-}^{\prime}\left(f\left(t_{1}\right), g\left(t_{1}\right)+h\left(t_{1}\right)\right)\right| \leq\left\|f\left(t_{1}\right)\right\| \cdot\left\|g\left(t_{1}\right)+h\left(t_{1}\right)\right\| \leq 2\|f\|_{\infty}^{2} .
\end{aligned}
$$

Combining (3.1), (3.17) and (3.18) and the equality $M(f)=\left\{t_{1}, t_{2}\right\}$, we immediately get $\rho_{+}^{\prime}(f, g+h)=2\|f\|_{\infty}^{2}, \quad \rho_{-}^{\prime}(f, g+h)=\eta$, and therefore

$$
\rho^{\prime}(f, g+h)=\frac{1}{2}\left(2\|f\|_{\infty}^{2}+\eta\right)=\|f\|_{\infty}^{2}+\frac{1}{2} \eta .
$$

Then, by (3.16), (3.19) and the assumption $f \in \mathcal{N}_{s m}^{s}\left(\mathcal{C}_{0}(\mathcal{T}, E)\right)$ we have

$$
\|f\|_{\infty}^{2}+\frac{1}{2}(\beta+\delta)=\rho^{\prime}(f, g)+\rho^{\prime}(f, h)=\rho^{\prime}(f, g+h)=\|f\|_{\infty}^{2}+\frac{1}{2} \eta,
$$

whence $\eta=\beta+\delta$. Using (3.12), (3.13), (3.14), (3.17) and (nd2) we have

$$
\begin{aligned}
& \rho_{-}^{\prime}\left(f\left(t_{1}\right), y+z\right) \quad \stackrel{(3.12)}{=} \quad \rho_{-}^{\prime}\left(f\left(t_{1}\right), \frac{1}{\|f\|_{\infty}} g\left(t_{1}\right)+\frac{1}{\|f\|_{\infty}} h\left(t_{1}\right)\right) \\
& \stackrel{(3.17)}{=} \quad \frac{1}{\|f\|_{\infty}} \eta=\frac{1}{\|f\|_{\infty}} \beta+\frac{1}{\|f\|_{\infty}} \delta \\
& \stackrel{(3.13),(3.14)}{=} \rho_{-}^{\prime}\left(f\left(t_{1}\right), \frac{1}{\|f\|_{\infty}} g\left(t_{1}\right)\right)+\rho_{-}^{\prime}\left(f\left(t_{1}\right), \frac{1}{\|f\|_{\infty}} h\left(t_{1}\right)\right) \\
& \stackrel{(3.12)}{=} \quad \rho_{-}^{\prime}\left(f\left(t_{1}\right), y\right)+\rho_{-}^{\prime}\left(f\left(t_{1}\right), z\right) \text {. }
\end{aligned}
$$

It follows from Lemma 3.6 that $f\left(t_{1}\right) \in \mathcal{N}_{s m}(E)$. In a similar way, one checks that $f\left(t_{2}\right) \in \mathcal{N}_{s m}(E)$. Therefore, we may say that the proof of Theorem 3.7 is complete.

Combining Theorems 3.3, 3.5 and 3.7, we immediately get the following characterizations.

Theorem 3.8. Suppose that $\mathcal{T}$ is a locally compact metric space and let $E$ be a normed space. Assume that $f \in \mathcal{C}_{0}(\mathcal{T}, E), f \neq 0$, and $f(t)$ is a smooth point for every $t \in \mathcal{M}(f)$. The following statements are equivalent:

(A) $f$ is a semi-smooth point, i.e., $f \in \mathcal{N}_{s m}^{s}\left(\mathcal{C}_{0}(\mathcal{T}, E)\right)$,

(B) $\quad \operatorname{card} \mathcal{M}(f) \leq 2$.

Theorem 3.9. Suppose that $\mathcal{T}$ is a locally compact metric space. Let $E$ be a smooth normed space. Assume that $f \in \mathcal{C}_{0}(\mathcal{T}, E), f \neq 0$. The following statements are equivalent:

(A) $f$ is a semi-smooth point, i.e., $f \in \mathcal{N}_{s m}^{s}\left(\mathcal{C}_{0}(\mathcal{T}, E)\right)$,

(B) $\operatorname{card} \mathcal{M}(f) \leq 2$. 
In this part of the section, we present another application of our main results in the classical sequence spaces, i.e., $c_{0}$ and $c$.

The sequence $x=\left(x_{1}, x_{2}, \ldots\right) \in c_{0}$ can be identified with a function $f_{x}: \mathbb{N} \rightarrow \mathbb{R}$ by $f_{x}(n):=x_{n}$. Clearly, $f_{x} \in \mathcal{C}_{0}(\mathbb{N}, \mathbb{R})$ and such an identification is a linear and topological isomorphism, which is also an isometry. In the same manner we can see that the space $c$ is identified with $\mathcal{C}(\mathbb{N} \cup\{+\infty\}, \mathbb{R})$, and in particular $\mathcal{C}(\mathbb{N} \cup\{+\infty\}, \mathbb{R})=\mathcal{C}_{0}(\mathbb{N} \cup\{+\infty\}, \mathbb{R})$.

Theorem 3.10. Suppose that $x=\left(x_{1}, x_{2}, \ldots\right) \in c_{0}$ and $x \neq 0$. Then $x$ is a semi-smooth point, i.e., $x \in \mathcal{N}_{s m}^{s}\left(c_{0}\right)$, if and only if one of the following conditions holds:

(a) $\exists_{k, \ell \in \mathbb{N}} \forall_{n \in \mathbb{N} \backslash\{k, \ell\}}:\left|x_{n}\right|<\left|x_{k}\right|=\left|x_{\ell}\right|=\|x\|_{\infty}$,

(b) $\exists !_{k \in \mathbb{N}} \forall_{n \neq k}:\left|x_{n}\right|<\left|x_{k}\right|=\|x\|_{\infty}$.

Proof. We can make the identification $f_{x} \in \mathcal{C}_{0}(\mathbb{N}, \mathbb{R})$ by $f_{x}(n):=x_{n}$ Theorem 3.9 implies $f_{x}$ is semi-smooth if and only if $\operatorname{card} \mathcal{M}\left(f_{x}\right) \leq 2$, which translates to conditions (a) and (b).

Theorem 3.11. Suppose that $x=\left(x_{1}, x_{2}, \ldots\right) \in c$ and $x \neq 0$. Then $x$ is a semismooth point, i.e., $x \in \mathcal{N}_{s m}^{s}(c)$, if and only if one of the following conditions holds:

(a) $\exists_{k, l \in \mathbb{N}} \forall_{n \in \mathbb{N} \backslash\{k, l\}}:\left|x_{n}\right|<\left|x_{k}\right|=\left|x_{l}\right|=\|x\|_{\infty}$ and $\lim _{n \rightarrow+\infty} x_{n} \in\left(-\|x\|_{\infty},\|x\|_{\infty}\right)$,

(b) $\forall_{n \in \mathbb{N}}:\left|x_{n}\right|<\|x\|_{\infty}$ and $\lim _{n \rightarrow+\infty} x_{n}=\|x\|_{\infty}$ or $-\|x\|_{\infty}$,

(c) $\exists !_{k \in \mathbb{N}} \forall_{n \neq k}:\left|x_{n}\right|<\left|x_{k}\right|=\|x\|_{\infty}$ and $\lim _{n \rightarrow+\infty} x_{n} \in\left(-\|x\|_{\infty},\|x\|_{\infty}\right)$.

Proof. Applying Theorem 3.9 again, this time to the identification $c=\mathcal{C}(\mathbb{N} \cup$ $\{+\infty\}, \mathbb{R})$, we obtain the desired assertion.

Example 3.12. Consider the set $\mathcal{T}:=\Sigma_{2}=\{-1,1\}^{\mathbb{N}}$. By Tychonoff theorem, $\Sigma_{2}$ is a compact metric space with product topology given by

$$
d(x, y):=\sum_{j=0}^{+\infty} \frac{\left|x_{j}-y_{j}\right|}{2^{j}}
$$

where $x=\left(x_{j}\right)_{j \in \mathbb{N}}$ and $y=\left(y_{j}\right)_{j \in \mathbb{N}}$ are in $\Sigma_{2}$. Denote $1^{\infty}=(1,1,1, \ldots)$ and $-1^{\infty}=(-1,-1,-1, \ldots)$.

Fix a number $\alpha>0$ and take $f_{\alpha}: \Sigma_{2} \rightarrow \mathbb{R}$ given by the formula

$$
f_{\alpha}(x)=f_{\alpha}\left(x_{0}, x_{1}, \ldots\right):=\sum_{j=0}^{+\infty} \frac{x_{j}}{(j+1)^{\alpha}} .
$$

It is easy to verify that $f_{\alpha} \in \mathcal{C}_{0}\left(\Sigma_{2}, \mathbb{R}\right)$. Furthermore, $M\left(f_{\alpha}\right)=\left\{-1^{\infty}, 1^{\infty}\right\}$ and thus by Theorem 3.9 we conclude that $f_{\alpha}$ is a semi-smooth point in the space $\mathcal{C}_{0}\left(\Sigma_{2}, \mathbb{R}\right)$. 
Clearly, one can take any $f \in \mathcal{C}_{0}\left(\Sigma_{2}, \mathbb{R}\right)$ with the following representation: $f(x)=\sum_{j=0}^{+\infty} a_{j} x_{j}$ for some suitable sequence $\left(a_{j}\right)_{j \in \mathbb{N}}$ with $a_{j} \neq 0$ for all $j \in \mathbb{N}$ such that $f(x)$ converges for any $x \in \Sigma_{2}$. Then $\|f\|_{\infty}=\left\|f\left(\left(\operatorname{sgn}\left(a_{j}\right)\right)_{j \in \mathbb{N}}\right)\right\|=$ $\left\|f\left(-\left(\operatorname{sgn}\left(a_{j}\right)\right)_{j \in \mathbb{N}}\right)\right\|$ and $\|f(x)\|<\|f\|_{\infty}$ for $x \in \Sigma_{2} \backslash\left\{-\left(\operatorname{sgn}\left(a_{j}\right)\right)_{j \in \mathbb{N}}\right.$, $\left.\left(\operatorname{sgn}\left(a_{j}\right)\right)_{j \in \mathbb{N}}\right\}$. Thus such an $f$ is a semi-smooth point.

The above considerations can be rewritten as the following result.

Theorem 3.13. Every unconditionally convergent series is a semi-smooth point in $\mathcal{C}_{0}\left(\Sigma_{2}, \mathbb{R}\right)$.

Proof. The series $\sum_{j=0}^{+\infty} a_{j}$ is identified with $f\left(x_{0}, x_{1}, \ldots\right)=\sum_{j=0}^{+\infty} a_{j} x_{j}$. The rest is clear.

Let $X$ be a real normed linear space. Let $X \oplus_{\infty} \ldots \oplus_{\infty} X$ denote the direct sum of $X$ with itself $n$ times, and in a natural way we introduce the norm $\left\|\left(x_{1}, \ldots, x_{n}\right)\right\|_{\infty}:=\max \left\{\left\|x_{1}\right\|, \ldots,\left\|x_{n}\right\|\right\}$. Note that $X \oplus_{\infty} X$ is identified with $\mathcal{C}_{0}(\{1,2\}, X)$. As an immediate consequence of Theorems 3.7 and 3.9, we have the following.

Corollary 3.14. $X$ is a smooth space if and only if $X \oplus_{\infty} X$ is a semi-smooth space.

Proof. Assume that $X$ is a smooth space. Take any non-zero $f \in \mathcal{C}_{0}(\{1,2\}, X)$. Since $X \oplus_{\infty} X=\left\{(g(1), g(2)): g \in \mathcal{C}_{0}(\{1,2\}, X)\right\}$ and $\|(f(1), f(2))\|_{\infty}=$ $\max \{\|f(1)\|,\|f(2)\|\}$, we have card $\mathcal{M}(f) \leq 2$. Thus, by Theorem 3.9 we obtain that $f$ is a semi-smooth point.

Conversely, let $X \oplus_{\infty} X$ be semi-smooth. Fix $x \in X \backslash\{0\}$. We can find a non-zero $f \in \mathcal{C}_{0}(\{1,2\} ; X)$ such that $x=f(1)=f(2)$. Then $\operatorname{card} \mathcal{M}(f)=2$ and by Theorem 3.7, we obtain that $x=f(1)$ is a smooth point.

We may identify $X \oplus_{\infty} X \oplus_{\infty} X$ with a space $\mathcal{C}_{0}(\{1,2,3\}, X)$, and applying again Theorem 3.5 and using a similar argument as in the proof of the above corollary, we obtain

Corollary 3.15. Let $X$ be any nontrivial normed space. Then $X \oplus_{\infty} X \oplus_{\infty} X$ is not a semi-smooth space.

Bearing in mind that the space $l^{1}$ is semi-smooth, we have the following result.

Proposition 3.16. Let $X$ be any nontrivial normed space. Then, there is no linear isometry $\mu$ from $X \oplus_{\infty} X \oplus_{\infty} X$ into $l^{1}$. 


\section{Semi-smooth Points in Normed Spaces and Supporting Functionals}

The condition (2.3) motivates this section. When $x_{o}$ is not a smooth point, consider the set $J\left(x_{o}\right)$. An interesting question in analysis is to study points for which $J\left(x_{o}\right)$ is a "large set". The question is: What about spaces which are not smooth? Motivated by these, in this section we prove some characterization of semi-smoothness (instead of smoothness).

Let us fix $D \subseteq X$ and $a \in D$. We say that $D$ is symmetric (with respect to the point $a$ ) if for all $x \in D$ we have $2 a-x \in D$ or, equivalently, $D=2 a-D$.

Theorem 4.1. Let $(X,\|\cdot\|)$ be a real normed linear space and $x_{o} \in X \backslash\{0\}$. Then the following statements are equivalent:

(A) $X$ is semi-smooth in $x_{o}$;

(B) $J\left(x_{o}\right)$ is symmetric with respect to some point $m^{*}$. Moreover, $\rho^{\prime}\left(x_{o}, y\right)=m^{*}(y)$ for $y \in X$.

Proof. Without loss of generality, we can assume that $\left\|x_{o}\right\|=1$ (see Theorem $2.3,(\mathrm{a}) \Leftrightarrow(\mathrm{e}))$. We start with proving $(\mathrm{B}) \Rightarrow(\mathrm{A})$. Fix $y \in X$. By $(2.2)$ we obtain

$$
x^{*}(y) \leq \rho_{+}^{\prime}\left(x_{o}, y\right) \text { for all } x^{*} \in J\left(x_{o}\right) .
$$

It yields

$$
\forall_{x^{*} \in J\left(x_{o}\right)} \quad 2 m^{*}(y)-\rho_{+}^{\prime}\left(x_{o}, y\right) \leq 2 m^{*}(y)-x^{*}(y) .
$$

It follows from $(\mathrm{B})$ that $2 m^{*}-x^{*} \in J\left(x_{o}\right)$ and $J\left(x_{o}\right)=2 m^{*}-J\left(x_{o}\right)$. Now the condition (4.1) becomes (putting $z^{*}:=2 m^{*}-x^{*}$ in place of $2 m^{*}-x^{*}$ in the above inequality)

$$
\forall_{z^{*} \in J(x)} \quad 2 m^{*}(y)-\rho_{+}^{\prime}\left(x_{o}, y\right) \leq z^{*}(y) .
$$

Passing to the infimum over $z^{*} \in J(x)$ (and applying Theorem 2.1) we get

$$
2 m^{*}(y)-\rho_{+}^{\prime}\left(x_{o}, y\right) \leq \rho_{-}^{\prime}\left(x_{o}, y\right) .
$$

It follows from the above inequality that $m^{*}(y) \leq \frac{1}{2} \rho_{-}^{\prime}\left(x_{o}, y\right)+\frac{1}{2} \rho_{+}^{\prime}\left(x_{o}, y\right)=$ $\rho^{\prime}\left(x_{o}, y\right)$. Thus we have

$$
\forall y \in X \quad m^{*}(y) \leq \rho^{\prime}\left(x_{o}, y\right) .
$$

Putting $-y$ in place of $y$ in the above inequality we get $m^{*}(-y) \leq \rho^{\prime}\left(x_{o},-y\right)$. Using (Msip2), we get $m^{*}(y) \geq \rho^{\prime}\left(x_{o}, y\right)$, which means

$$
\forall y \in X \quad m^{*}(y) \geq \rho^{\prime}\left(x_{o}, y\right) .
$$

Inequalities (4.2) and (4.3) yield $\forall_{y \in X} m^{*}(y)=\rho^{\prime}\left(x_{o}, y\right)$. Thus finally we get $\rho^{\prime}\left(x_{o}, \cdot\right) \in X^{*}$. That means $x_{o} \in \mathcal{N}_{s m}^{s}(X)$ (see Theorem 2.3).

We prove the converse implication. If we assume $(\mathrm{A})$, then $\rho^{\prime}\left(x_{o}, \cdot\right) \in X^{*}$. By (Msip3) and (Msip4) we get $\rho^{\prime}\left(x_{o}, \cdot\right) \in J\left(x_{o}\right)$. Let $m^{*} \in X^{*}$ be defined by $m^{*}(\cdot):=\rho^{\prime}\left(x_{o}, \cdot\right)$. We show that $J\left(x_{o}\right)$ is symmetric. Fix $z^{*} \in J\left(x_{o}\right)$. Therefore, 
$2 m^{*}\left(x_{o}\right)-z^{*}\left(x_{o}\right)=2 \rho^{\prime}\left(x_{o}, x_{o}\right)-\left\|x_{o}\right\|=2\left\|x_{o}\right\|^{2}-\left\|x_{o}\right\|=2 \cdot 1-1=\left\|x_{o}\right\|$. Next we prove that $\left\|2 m^{*}-z^{*}\right\|=1$. Fix $y \in S(X)$ and notice that we have

$$
\begin{aligned}
2 m^{*}(y)-z^{*}(y) & =2 \rho^{\prime}\left(x_{o}, y\right)-z^{*}(y)=\rho_{-}^{\prime}\left(x_{o}, y\right)+\rho_{+}^{\prime}\left(x_{o}, y\right)-z^{*}(y) \\
& \stackrel{(2.2)}{\geq} \rho_{-}^{\prime}\left(x_{o}, y\right)+\rho_{+}^{\prime}\left(x_{o}, y\right)-\rho_{+}^{\prime}\left(x_{o}, y\right)=\rho_{-}^{\prime}\left(x_{o}, y\right) \stackrel{(\text { nd } 5)}{\geq}-1
\end{aligned}
$$

and

$$
\begin{aligned}
2 m^{*}(y)-z^{*}(y) & =2 \rho^{\prime}\left(x_{o}, y\right)-z^{*}(y)=\rho_{-}^{\prime}\left(x_{o}, y\right)+\rho_{+}^{\prime}\left(x_{o}, y\right)-z^{*}(y) \\
& \stackrel{(2.2)}{\leq} \rho_{-}^{\prime}\left(x_{o}, y\right)+\rho_{+}^{\prime}\left(x_{o}, y\right)-\rho_{-}^{\prime}\left(x_{o}, y\right)=\rho_{+}^{\prime}\left(x_{o}, y\right) \stackrel{(\text { nd5 })}{\leq} 1 .
\end{aligned}
$$

It follows from the above inequalities that $\left|2 m^{*}(y)-z^{*}(y)\right| \leq 1$ for all $y \in S(X)$. We know that $2 m^{*}\left(x_{o}\right)-z^{*}\left(x_{o}\right)=1$ and $\left\|x_{o}\right\|=1$. Therefore $\left\|2 m^{*}-z^{*}\right\|=1$, and hence $2 m^{*}-z^{*} \in J\left(x_{o}\right)$.

As a consequence of the above theorem, we get the following result.

Corollary 4.2. Let $(X,\|\cdot\|)$ be a real normed linear space and $x_{o} \in X \backslash\{0\}$. If $J\left(x_{o}\right)$ is a segment, i.e., $J\left(x_{o}\right)=\operatorname{conv}\left\{x^{*}, y^{*}\right\}$, then $X$ is semi-smooth at $x_{o} \in S_{X^{*}}$. Moreover, $\rho^{\prime}\left(x_{o}, \cdot\right)=\frac{1}{2} x^{*}(\cdot)+\frac{1}{2} y^{*}(\cdot)$.

Fix a nonzero $x \in X$. If $\operatorname{dim} X=2$, then $\operatorname{dim} X^{*}=2$. Since $J(x)$ is a convex and $J(x) \subseteq S\left(X^{*}\right)$, the equality $\operatorname{dim} X^{*}=2$ shows that $\operatorname{card} J(x)=1$ or $J(x)=\operatorname{conv}\left\{x^{*}, y^{*}\right\}$. Hence $x \in \mathcal{N}_{s m}(X)$ or $x \in \mathcal{N}_{s m}^{s}(X)$. As an immediate consequence of this reasoning, we have the following.

Corollary 4.3. Every two-dimensional normed space is semi-smooth.

\section{Concluding Remarks}

If $X$ is a Banach space that is separable, then $\mathcal{N}_{s m}(X)$ and $\mathcal{N}_{s m}^{s}(X)$ must be dense. We know that $\mathcal{N}_{s m}(X) \subseteq \mathcal{N}_{s m}^{s}(X)$. What does this say about $\mathcal{N}_{s m}^{s}(X) \backslash$ $\mathcal{N}_{s m}(X)$ ? We have already seen that

$$
\mathcal{N}_{s m}^{s}(\mathcal{C}[0,1]) \mathcal{N}_{s m}(\mathcal{C}[0,1])=\{f \in \mathcal{C}[0,1]: \operatorname{card} \mathcal{M}(f)=2\} .
$$

Now, let $\mathcal{H}$ be a real Hilbert space and let $\mathcal{K}(\mathcal{H})$ be a space of compact linear operators. The following result was obtained in [14]:

$$
\mathcal{N}_{\text {sm }}^{s}(\mathcal{K}(\mathcal{H})) \backslash \mathcal{N}_{\text {sm }}(\mathcal{K}(\mathcal{H}))=\{A \in \mathcal{K}(\mathcal{H}): \operatorname{dim} \operatorname{span} M(A)=2\},
$$

where $M(A):=\left\{x \in S_{\mathcal{H}}:\|A x\|=\|A\|\right\}$. It is easy to check that the above two sets, i.e., (5.1) and (5.2), are dense and nowhere dense, respectively.

It is known that

$$
x \in \mathcal{N}_{s m}\left(c_{0}\right) \Leftrightarrow \exists !_{k \in \mathbb{N}} \forall_{n \neq k}:\left|x_{n}\right|<\left|x_{k}\right|=\|x\|_{\infty} .
$$


Therefore, $\mathcal{N}_{s m}\left(c_{0}\right)$ is easily seen to be open. But since $\mathcal{N}_{s m}\left(c_{0}\right)$ is dense, $\mathcal{N}_{s m}^{s}\left(c_{0}\right) \backslash \mathcal{N}_{s m}\left(c_{0}\right)$ is nowhere dense. This is summarized as follows.

$$
\begin{array}{|c||c|c|c|}
X & \mathcal{N}_{s m}(X) & \mathcal{N}_{s m}^{s}(X) & \mathcal{N}_{s m}^{s}(X) \backslash \mathcal{N}_{s m}(X) \\
---- & ------- & ---- & -------- \\
\mathcal{K}(\mathcal{H}) & \text { dense } & \text { dense } & \text { nowhere dense } \\
\mathcal{C}[0,1] & \text { dense } & \text { dense } & \text { dense } \\
c_{0} & \text { dense and open } & \text { dense } & \text { nowhere dense } \\
\mathcal{H} & \mathcal{H} & \mathcal{H} & \emptyset
\end{array}
$$

It is amazing that the sets $\mathcal{N}_{s m}^{s}, \mathcal{N}_{s m}$ are always huge but the set $\mathcal{N}_{s m}^{s} \mathcal{N}_{s m}$ may be large or small.

We end this work with an inquisitive question. Is it possible to find a normed linear space $X$ such that $\operatorname{dim} X \geq 3$ and $\mathcal{N}_{s m}(X)=\mathcal{N}_{s m}^{s}(X) \neq X$ ?

\section{Funding None.}

\section{Declarations}

Conflict of interest The authors declare that they have no conflicts of interest.

Availability of Data and Material Not applicable.

Code Availability Not applicable.

Open Access. This article is licensed under a Creative Commons Attribution 4.0 International License, which permits use, sharing, adaptation, distribution and reproduction in any medium or format, as long as you give appropriate credit to the original author(s) and the source, provide a link to the Creative Commons licence, and indicate if changes were made. The images or other third party material in this article are included in the article's Creative Commons licence, unless indicated otherwise in a credit line to the material. If material is not included in the article's Creative Commons licence and your intended use is not permitted by statutory regulation or exceeds the permitted use, you will need to obtain permission directly from the copyright holder. To view a copy of this licence, visit http://creativecommons. org/licenses/by/4.0/.

\section{References}

[1] Alsina, C., Sikorska, J., Tomás, M.S.: Norm Derivatives and Characterizations of Inner Product Spaces. World Scientific, Hackensack (2010)

[2] Banach, S.: Theorie des operationes lineaires, Warszawa (1932)

[3] Chmieliński, J., Sikorska, J., Wójcik, P.: On a $\rho$-orthogonally additive mappings. Results Math. 75, 108 (2020)

[4] Chmieliński, J., Wójcik, P.: On a $\rho$-orthogonality. Aequ. Math. 80, 45-55 (2010)

[5] Chmieliński, J., Wójcik, P.: $\rho$-orthogonality and its preservation-revisited. Banach Center Publications 99, 17-30 (2013) 
[6] Deville, R., Godefroy, G., Zizler, V.: Smoothness and Renormings in Banach Spaces. Harlow, Essex (1993)

[7] Dragomir, S.S.: Semi-inner Products and Applications. Nova Science, Hauppauge (2004)

[8] Miličić, P.M.: Sur le semi-produit scalaire dans quelques espaces vectorial normès. Mat. Vesnik 8(23), 181-185 (1971)

[9] Miličić, P.M.: Sur la G-orthogonalité dans les espaces normés. Mat. Vesnik 39, 325-334 (1987)

[10] Singer, I.: Sur la meilleure approximation des fonctions abstracts continues á valeurs dans un espace de Banach. Riv. Mat. Pura Appl. 11, 245-262 (1957)

[11] Sunderesan, K.: Some geometric properties of the unit cell in spaces C(X, B). Bull. Polish Acad. Sci. (Math.) XIX(11), 1007-1012 (1971)

[12] Wójcik, P.: Characterization of linear similarities through functional equation and mappings preserving orthogonalities. Linear Algebra Appl. 579, 206-216 (2019)

[13] Wójcik, P.: On geometry of Banach function modules - selected topics. In: Chapter 20, Ulam's Type Stability. Springer, pp. 453-468 (2019)

[14] Wójcik, P.: Semi-smooth points in space of operators on Hilbert space. Oper. Matrices 14, 951-958 (2020)

Beata Derȩgowska, Beata Gryszka, Karol Gryszka and Paweł Wójcik

Institute of Mathematics

Pedagogical University of Kraków

Podchorążych 2

Kraków

Poland

e-mail: beata.deregowska@up.krakow.pl;

beata.gryszka@up.krakow.pl;

karol.gryszka@up.krakow.pl;

pawel.wojcik@up.krakow.pl

Received: November 15, 2020.

Accepted: October 19, 2021.

Publisher's Note Springer Nature remains neutral with regard to jurisdictional claims in published maps and institutional affiliations. 